\title{
Mutation watch:
}

\section{PEX PLUS? Gene(s) for X-linked hypophosphatemia and deafness}

\section{Miriam Meisler}

\begin{abstract}
Department of Human Genetics, 4708 Medical Science II, University of Michigan, Ann Arbor, Michigan 48109-0618, USA
\end{abstract}
Received: 4 April 1997 / Accepted: 17 April 1997

Three decades of work on X-linked hypophosphatemia in human and mouse reached fruition with the recent discovery of PEX, the "Phosphate regulating gene with homologies to Endopeptidases on the X chromosome" (HYP Consortium 1995; Strom et al. 1997). The mouse story began in 1966, when six males with shortened trunk and hindlimbs were discovered during a linkage experiment at The Jackson Laboratory (Eicher et al. 1976). Biochemical detective work identified hypophosphatemia as a critical defect, and administration of phosphate to the drinking water corrected many of the skeletal abnormalities and the dwarfing. Abnormal renal phosphate reabsorption accounts for the low level of phosphate in blood and the elevated level in urine. The mutation was inherited as an X-linked dominant and designated hypophosphatemia (Hyp).

In 1986, a second dominant X-linked hypophosphatemia mutation, gyro $(G y)$, was described (Lyon et al. 1986). In addition to hypophosphatemia and skeletal defects that closely resemble Hyp, gyro mice exhibit circling behavior due to degeneration of hair cells in the vestibular apparatus and cochlea. The neuroepithelial abnormalities of the inner ear also result in a hearing deficiency (Steel 1995). Because the phenotypes of Hyp and Gy are dominant, it was not possible to draw conclusions about possible allelism from the phenotype of the compound heterozygote. Evidence for very close linkage was obtained from a cross between $G y+/+$ Hyp females and normal males (Lyon et al. 1986). Among the 239 male offspring of this cross, one had normal blood phosphate levels and generated normal male offspring, indicating that he carried a recombinant ++ chromosome. This recombination was interpreted as evidence that $H y p$ and $G y$ were mutations in distinct but closely linked and functionally related genes. However, recent molecular analysis found that Hyp and Gy are caused by independent mutations in the Pex gene that are separated by approximately $100 \mathrm{~kb}$ (Strom et al. 1997). The original recombination between $G y$ and Hyp was thus a rare example of intragenic recombination and reminds us that a single recombination does not provide a reliable estimate of genetic distance.

\section{Positional cloning of human PEX}

Human X-linked hypophosphatemia, or Vitamin D-resistant rickets (MIM 307800), is a dominant disorder with an incidence of $1 / 20,000$ characterized by a renal phosphate transport defect, hypophosphatemia, and elevated urinary phosphate. High-resolution genetic mapping was achieved by analysis of 258 family members from 20 kindreds (Rowe et al. 1996). A YAC contig spanning 3.3 $\mathrm{Mb}$ was generated, and an interesting application of the Southwestern blotting technique demonstrated the presence of Vitamin $\mathrm{D}$ receptor binding sites in one YAC that subsequently proved to contain the disease gene. Southern blotting of genomic DNA from 150 families and hybridization with radiolabeled cosmid DNA detected deletions in four families (HYP Consortium 1995). Cosmids from the deleted region were sequenced and used for cDNA selection, resulting in a cDNA contig that overlapped the patient deletions. Database sequence comparisons demonstrated that the new gene, PEX, is a member of a subfamily of endopeptidases involved in activation and degradation of a variety of peptide hormones. Other family members include neutral endopeptidase (NEP), endothelin-converting enzyme 1 (ECE-1) and the Kell antigen. One interesting feature of PEX and related peptidases is the presence of a putative transmembrane domain near the $\mathrm{N}$-terminus of the protein.

Subsequent isolation and sequencing of the mouse Pex cDNA revealed a predicted protein of 749 amino acids with $96 \%$ sequence identity to the human protein (Du et al. 1996; Strom et al. 1997). Northern blotting demonstrated a 6.6-kb transcript in calvaria and osteoblasts that was absent from Hyp tissues (Du et al. 1996). Detection of a low level of Pex transcript by RT-PCR suggested the possibility of a regulatory mutation in Hyp. However, when Pex cDNA fragments were used as probes on Southern blots of genomic DNA, large deletions were identified in both Hyp and $G y$ (Strom et al. 1997). The mutations are located at opposite ends of the cDNA, with loss of exons 1-3 in $G y$ and loss of exons 15-22 in Hyp. The gyro deletion was induced by X-radiation, but Hyp was a spontaneous mutation.

Although mutations in PEX have now been clearly associated with hypophosphatemia in human and mouse, the biochemical mechanism responsible for the clinical phenotypes remains unclear. One hypothesis is that PEX may be involved in cleavage of an as yet unidentified polypeptide hormone involved in phosphate transport. In addition to the medical benefits, the effort involved in positional cloning of HYP has been rewarded by the discovery of a novel metabolic pathway of fundamental biological importance.

\section{A new deafness gene?}

Also remaining for future investigation are the inner ear defects in gyro mice that are also seen in some human patients with HYP. It has been suggested that the abnormal phosphate metabolism itself could be directly or indirectly responsible. The identification of the gyro deletion, which extends for $\leq 40 \mathrm{~kb}$ upstream of Pex, raises the alternative possibility that a second deleted gene could account for the inner ear phenotype (Strom et al. 1997). Consistent with this hypothesis, a human nonsyndromic deafness disorder (DFN6) was recently mapped to Xp22 close to PEX (del Castillo et al. 1996). In view of the genetic and molecular resources now available, we may expect the rapid identification of the locus or loci responsible for this inner ear disorder.

\section{References}

del Castillo I, Villamar M, Sarduy M, Romero L, Herraiz C, Hernandez FJ, Rodriguez M, Borras I, Montero A, Bellon J, Tapia MC, Moreno F (1996) A novel locus for non-syndromic sensorineural deafness (DFN6) maps to chromosome Xp22. Hum Mol Genet 5, 1383-1387 
Du L, Desbarats M, Viel J, Glorieux FH, Cawthorn C, Ecarot B (1996) cDNA cloning of the murine Pex gene implicated in X-linked hypophosphatemia and evidence for expression in bone. Genomics 36, 22-28

Eicher EM, Southard JL, Scriver CR, Glorieux GH (1976) Hypophosphatemia: mouse model for human familial hypophospathemic (vitamin D-resistant) rickets. Proc Natl Acad Sci USA 73, 4667-4671

HYP Consortium (1995) A gene (PEX) with homologies to endopeptidases is mutated in patients with $\mathrm{X}$-linked hypophosphatemic rickets. Nature Genet 11, 130-136

Lyon MF, Scriver CR, Baker LRI, Tenenhouse HS, Kronick J, Mandla S (1986) The Gy mutation: another cause of X-linked hypophosphatemia in mouse. Proc Natl Acad Sci USA 83, 4899-4903
Rowe PSN, Goulding JN, Francis F, Oudet C, Econs MJ, Hanauer A, Lehrach H, Read AP, Mountford RC, Summerfield T, Weissenbach J, Fraser W, Drezner MK, Davies KE, O'Riordan JLH (1996) The gene for $\mathrm{X}$-linked hypophosphatemic rickets maps to a $200-300 \mathrm{~kb}$ region in $\mathrm{Xp} 22.1$, and is located on a single YAC containing a putative vitamin D response element (VDRE). Hum Genet 97, 345-352

Steel KP (1995) Inherited hearing defects in mice. Annu Rev Genet 29, 675-701

Strom TM, Francis F, Lorenz B, Böddrich A, Econs MJ, Lehrach H, Meitinger T (1997) Pex gene deletions in Gy and Hyp mice provide mouse models for X-linked hypophosphatemia. Hum Mol Genet 6, 165171 